\title{
A Fast Exponential Calculation for the IBM 370-195 at the Oak Ridge Gaseous Diffusion Plant
}

\author{
J. C. Whitson \\ J. H. Whealton
}

MASTER

\section{OAK RIDGE NATIONAL LABORATORY}




\section{DISCLAIMER}

This report was prepared as an account of work sponsored by an agency of the United States Government. Neither the United States Government nor any agency Thereof, nor any of their employees, makes any warranty, express or implied, or assumes any legal liability or responsibility for the accuracy, completeness, or usefulness of any information, apparatus, product, or process disclosed, or represents that its use would not infringe privately owned rights. Reference herein to any specific commercial product, process, or service by trade name, trademark, manufacturer, or otherwise does not necessarily constitute or imply its endorsement, recommendation, or favoring by the United States Government or any agency thereof. The views and opinions of authors expressed herein do not necessarily state or reflect those of the United States Government or any agency thereof. 


\section{DISCLAIMER}

Portions of this document may be illegible in electronic image products. Images are produced from the best available original document. 


\section{Printed in the United States of America. Available from}

National Technical Information Service

U.S. Department of Commerce

5285 Port Royal Road, Springfield, Virginia 22161

Price: Printed Copy $\$ 3.50 ;$ Microfiche $\$ 3.00$

This report was prepared as an account of work sponsored by the United States Government. Neither the United States nor the Energy Research and Development Administration/United States Nuclear Regulatory Commission, nor any of their employees, nor any of their contractors, subcontractors, or their employees, makes any warranty, express or implied, or assumes any legal liability or responsibility for the accuracy. completeness or usefulness of any information, apparatus, product or process disclosed, or represents that its use would not infringe privately owned rights. 
Contract No. W-7405-eng-26

Fusion Energy Division

A FAST EXPONENTIAL CALCULATION FOR THE IBM 370-195

AT THE OAK RIDGE GASEOUS DIFFUSION PLANT

J. C. Whitson and J. H. Whealton

Date Published: January 1977

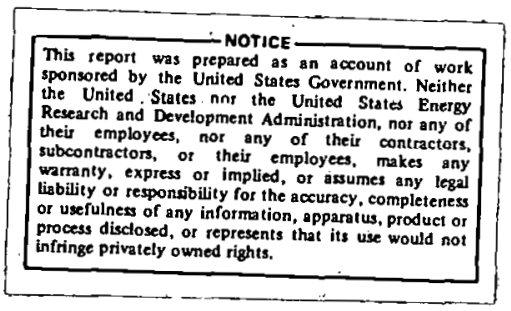

NOTICE This document contains information of a preliminary nature.

It is subject to revision or correction and therefore does not represent a final report.

\author{
Prepared by the \\ OAK RIDGE NATIONAL LABORATORY \\ Oak Ridge, Tennessee 37830 \\ operated by \\ UNION CARBIDE CORPORATION \\ for the \\ ENERGY RESEARCH \& DEVELOPMENT ADMINISTRATION
}


A FAST EXPONENTIAL CALCULATION FOR THF IRM 370-195

AT THE OAK RIDGE GASEOUS DIFFUSION PLANT

J. C. Whitson and J. H. Whealton

Oak Ridge National Laboratory

Oak Ridge, Tennessee

\begin{abstract}
The time spent for computing an exponential for the IBM 370-195 at the Oak Ridge Gaseous Diffusion Plant is about 25 times slower than that spent by the CDC-7600 CTR computer at Livermore. We propose a scheme which does not involve a function call, is 15 times faster than the IBM 370-195 scheme, and is useful when the exponential is called many times.
\end{abstract}


We propose here a scheme - which is 15 times faster than that presently used, does not involve a function call, and proves useful when the exponential is computed many times - for computing an exponential for the IBM 370-195 at the Oak Ridge Gaseous Diffusion Plant.

First a table of 341 values is computed. (once for the entire program) using the standard IBM function calls in the main program:

COMMON/EXPCOM/EXX(341)

$$
\begin{aligned}
& \text { IEXP }=-171 \\
& D 01 I=1,341 \\
& \text { IEXP }=\text { IEXP }+1 \\
& X X X P=I E X P \\
& \operatorname{EXX(I)~}=\operatorname{EXP(XEXP)}
\end{aligned}
$$

1 CONTINUE;

in the subroutine where the exponential is called repeatedly. A Taylor's series expansion about the near integer is performed:

$$
e^{x}=e^{a}\left\{1+(x-a)+\frac{(x-a)^{2}}{2 !}+\frac{(x-a)^{3}}{3 !}+\ldots\right\} \text {. }
$$

Specifically, the following will suffice in IBM single prectsion:

COMMON/EXPCOM/EXX(341)

$$
\begin{aligned}
& \text { PEX(Z) }=\left(1+Z\left(1+\frac{Z}{2}\left(1+\frac{z}{3}\left(1+\frac{Z}{4}\left(1+\frac{z}{5}\left(1+\frac{z}{6}\left(1+\frac{z}{7}\left(1+\frac{z}{8}\right)\right)\right)\right)\right)\right)\right)\right) \\
& U=\text { ARGEXP }+.500001 \\
& \text { IF (ARGEXP }<0) U=\text { ARGEXP }-.500001 \\
& I U=U \\
& \text { LUP }=\text { IU + 171 } \\
& \text { IF (LUP }<0) \text { GO TO } 2
\end{aligned}
$$




\author{
IF (LUP > 34I) STOP \\ $X U=I U$ \\ $X N A=A R G E X P-X U$ \\ $\operatorname{EXPP}=\operatorname{EXX}(L U P) \star P E X(X N A)$ \\ 3 CONTINUE \\ $2 \quad$ EXPP $=0$ \\ GO TO 3.
}




\section{THIS PAGE}

\section{WAS INTENTIONALLY \\ LEFT BLANK}


INTERNAL DISTRIBUTION ORNL/TM-5719

i. G. C. Barber

2. L. A. Berry

3. J. D. Callen

4. J. F. Clarke

5. W. K. Dagenhart

6. R. A. Dandl

7. R. C. Davis

8. C. A. Foster

9. W. L. Gardner

10. R. L. Grisham

11. H. H. Haselton

12. P. N. Haubenreich

13. T. C. Jernigan

14. G. G. Kelley

15. J. Kim

16. J. F. Lyon

17. F. B. Marcus

18. D. G. McAlees

19. S. L. Milora

20. 0. B. Morgan
21. N. S. Ponte

22. M. Roberts

23. J. A. Rome

24. M. W. Rosenthal

25. P. M. Ryan

26. D. E. Schechter

27. G. Schilling

28. D. Steiner

29. L. D. Stewart

30. W. L. Stirling

31. C. C. Tsai

32-42. J. H. Whedl LuII

43-53. J. C. Whitson

54. R. E. Wright

55-56. Central Research Library

57. Document Reference Section

58-59. Laboratory Records

60. Laboratory Records, ORNL-RC

61. ORNL Patent Office

62-63. Fusion Energy Division Library

\section{EXTERNAL DISTRIBUTION}

64. J. W. Beal, Development \& Technology, Division of Magnetic Fusion Energy, Energy Research \& Development Administration, Washington, DC 20545.

65. Dr. Klaus Berkner, University of California, Lawrence Berkeley Laboratory, Berkeley, CA 94720.

66. Dr. A. Cole, Culham Laboratory, UKAEA Research Group, Abingdon, Oxfordshire, 0X14 3DB England.

67. Dr. W. S. Cooper, University of California, Lawrence Berkeley Laboratory, Berkeley, CA 94720.

68. S. 0. Dean, Assistant Director, Office of Confinement Systems, Division of Magnetic Fusion Energy, Energy Research \& Development Administration, Washinytun, DC 20545.

69. Harold K. Forsen, Exxon Nuclear Co., Inc., 777 106th Ave., NE, Dellevue, WA 98004.

70. T. K. Fowler, University of California, Lawrence Livermore Laboratory, P.0. Box 808, Livermore, CA 94551. 
71. H. P. Furth, Plasma Physics Laboratory, Princeton University, Princeton, NJ 08540.

72. Dr. C. Geller, CEA - Centre d'Etudes Nucleaire, Division of Plasma Physics, Grenoble, France.

73. Dr. T. S. Green, Culham Laboratory, UKAEA Research Group, Abingdon, Oxfordshire, 0x14 30B England.

74. E. E. Kintner, Director, Division of Magnetic Fusion Energy, Energy Research \& Development Administration, Washington, DC. 20545.

75. Ur. E. H. Hooper, University of California, Lawrence Livermore Laboratory, P.0. Box 808, Livermore, CA 94550.

76. Dr. J. Junker, Max Planck Institut fur Plasmaphysik, Abteilung E2, 8046 Garching bei Munchen, Federal Republic of Germany.

77. Librarian, Culham Laboratory, UKAEA, Abingdon, Oxfordshire, $0 \times 14$ 3DB England.

78. Librarian, Institut fur Plasmaphysik, Abteilung E2, 8046 Garching bei Munchen, Federal Republic of Germany.

79. Plasma Physics Library, Plasma Physics Laboratory, Princeton University, P.0. Box 451, Princeton, NJ 08540.

80. Shinzaburo Matsuda, Thermonuclear Library, Japan Atomic Energy Research Institute, Tokai, Naka, Ibaraki, Japan.

81. T. Ohkawa, General Atomic Company, Inc., P.0. Box 608, San Diego, CA 92112 .

82. Dr. K. Prelec, Brookhaven National Laboratory, Upton, Long Island, NY 11973.

83. Dr. R. V. Pyle, University of California, Lawrence Berkeley Laboratory, Berkeley, CA 94720.

84. D. J. Rose, Department. of Nuclear Engineering, Masiachusctts Institute of Technology, Cambridge, MA 02139.

85. F. R. Scott, Electrical Power and Research Institute, 3412 Hillview Ave., P.0. Box 10412, Palo Alto, CA 94303.

86. N. N. Semashko, Kurchatov Atomic Energy Institute, Moscow, U.S.S.R.

87. Th. J. M. Sluyters, Brookhaven National Laboratory, Upton, Long Island, NY 11973. 
88. D. R. Sweetman, Culham Laboratory, UKAEA Research Group, Abingdon, Oxfordshire, 0X14 3DB England.

89. E. Thompson, Culham Laboratory, UKAEA Research Group, Abingdon, Oxfordshire, 0X14 3DB England.

90. V. M. Tuchevich (Att: V. E. Golant), A. F. Ioffe Physical Institute of the Academy of Sciences of the U.S.S.R., Leningrad K-21, U.S.S.R.

91-94. Dr. F. Valcx, DPH-PFC, Centre d'Etudes Nucleaire, BP No. 6, Fontenay-aux-Roses (Seine), France.

95. J. M. Williams, Assistant Director, Development and Technology, Division of Magnetic Fusion Energy, Energy Research \& Development Administration, Washingtur, DC 20545.

96. J. T. Woo, Massachusetts Institute of Technology, Rm. 38-176, Cambridge, MA 02139.

97. Dr. Herbert H. Woodson, Chairman, Department of Electrical Engineering, The University of Texas at Austin, Austin; TX 78712.

98. L. M. Branscomb, Vice President, IBM, Armonk, NY 10504.

99. S. B. Woo, Physics Department, University of Deleware, Newark, DE 19711.

100-126. Technical Information Center, ERDA, Oak Ridge, TN 37830.

127. Director, Research and Technical Support Div., ERDA-ORO. 